\title{
Cloud Removal in Image Time Series through Unmixing
}

\author{
Daniele Cerra, Rupert Müller, and Peter Reinartz \\ German Aerospace Center (DLR) \\ Remote Sensing Technology Institute (IMF) \\ 82234 Wessling, Germany \\ Email: daniele.cerra@dlr.de, rupert.mueller@dlr.de, peter.reinartz@dlr.de
}

\section{INTRODUCTION}

Satellite Image Time Series (SITS) are sets of images acquired by the same or different spaceborne sensors over the same area at different acquisition times. In recent years, SITS acquired by multispectral sensors have increased, due to the large availability of these kind of data and the decrease in revisit time. The latter aspect is also a consequence of the advent of multispectral satellite constellations such as RapidEye and Pleiades, with the Sentinel 2 mission being able to derive such products at global scale in the near future. The high dimensionality of SITS unlocks the use of methods usually restricted to the processing of hyperspectral images, typically characterized by hundreds of narrow, contiguous spectral bands. This paper proposes a new cloud removal algorithm for scenes within a SITS based on the concept of spectral unmixing, which decomposes hyperspectral image elements into fractional abundances of reference spectra related to the materials present in a given scene. In the case of SITS, we consider a reference spectrum as a pixel which presents a characteristic evolution in time of its spectral features. While traditional methods aim at substituting the affected pixels with other ones taken from other scenes, the proposed algorithm allows exploiting the full spectral and temporal information of the SITS to synthesize the expected values below a cloudy area. This is done independently from the overall atmospheric interactions affecting a given image, and it is possible even if only one acquisition is available for a given period of time.

\section{Hyperspectral Images and Spectral Unmixing}

Hyperspectral images are characterized by a high number of narrow, adjacent spectral bands often in the order of hundreds, while as a comparison a typical multispectral sensor has less than 10 (broader) bands. Several algorithms for hyperspectral image processing have been defined which cannot be adapted to be applied to other kinds of datasets, as they require a high dimensional space to operate. An example is spectral unmixing, which aims at decomposing each image element as a linear (or less often non-linear) combination of signals typically related to pure materials, often called endmembers [1]. These methods give as output abundances maps, which quantify the contribution of each endmember to a given pixel. Therefore a pixel $m$ could be expressed as:

$$
m=\sum_{i=1}^{k} x_{i} s_{i}+r,
$$

where $x_{1} \ldots x_{k}$ and $s_{1} \ldots s_{k}$ are the fractional abundances and the spectra of the $k$ available and pre-selected endmembers, while $r$ is a residual vector containing the portion of the signal which cannot be represented in terms of the basis vectors of choice. Therefore, if in a scene we have only mixtures of two materials in each pixel, for example water and soil, $m$ could be expressed as $m=x_{\text {water }} s_{\text {water }}+x_{\text {soil }} s_{\text {soil }}+r$.

Unmixing-based Denoising (UBD) exploits spectral unmixing results to selectively recover bands affected by a low Signal-to-Noise Ratio (SNR) in hyperspectral images [2]. The output of the described spectral unmixing process is inferred into the reconstruction of a given noisy band. By considering the physical properties of a mixed spectrum, UBD ignores the residual vector $r$ in the reconstruction, by assuming that $r$ is mostly composed by noise, and more relevant in spectral bands where atmospheric absorption effects are stronger. Each 
image element $m$ is then reconstructed as:

$$
\hat{m}=\sum_{i=1}^{k} x_{i} s_{i},
$$

ignoring $r$ in eq. (1), and along with it most of the noise affecting $m$.

\section{Spectral Unmixing for Cloud Removal in IMAGE TIME SERIES}

In [3], [4] UBD has been applied for the inpainting of missing values in single bands of hyperspectral images, outperforming algorithms based on spatial or spectral averaging of other pixels, smoothness of Partial Differential Equations, or reconstruction through Discrete Cosine Transform coefficients. In this case, the information inferred from all the available spectral bands was used to synthesize the missing or corrupted values as a linear combination of selected pixels in the same affected spectral band. This is possible only if the missing values are only to be found in few spectral bands, ideally one. In the case of SITS, pixels covered by clouds in a given image may be regarded as corrupted, as usually it is more of interest to measure the reflected solar radiation from the ground. It could be desirable then to perform inpainting of these image elements, by estimating their expected value. Unfortunately, unmixing-based methods such as UBD need to operate in a high dimensionality space. Namely, the number of dimensions should be reasonably higher than the so-called Virtual Dimensionality (VD) of the data, which is related to the number of different materials present in a given scene. Therefore, these methods are hard to apply in practice to multispectral datasets. Nevertheless, in the case of SITS the high dimensionality of a stack of multispectral images may be comparable to the one of a hyperspectral dataset. This allows applying methods defined for hyperspectral remote sensing to SITS. We proceed using a spectral unmixing-based method to remove clouds from a single image in a SITS as follows.

First of all, cloud and cloud shadows must be masked out. To achieve this, automatic algorithms such as [5] and [6] can be employed. Subsequently, representative cloud-free image elements must be located in the scene. For this purpose, the image stack can be fed to any hyperspectral endmember extraction algorithm such as N-Finder [7], while the VD can be previously estimated with an ad hoc algorithm of choice [1]. In this case, an "endmember" means an object which has a characteristic evolution in time, such as an agricultural field of some kind. A sample output of this process is reported in Fig. 1. As we are operating in a high-dimensional space, the selected pixels can be linearly combined to reconstruct the spectral and temporal information of any other pixel in the scene, minimizing the residual in eq. (1). Once the abundance vectors are estimated, these are used to reconstruct the affected pixels using eq. (2). The workflow is reported in Fig. 2.

The proposed method is similar to the Contextual Multiple Linear Prediction (CMLP) reconstruction method described in [8], in which corrupted values are predicted by linear combination of some preselected classes of interest chosen outside of the cloudy area, but the use of algorithms typically applied to hyperspectral data introduces some important differences with CMLP. While the latter performs an unsupervised classification of the image to extract reference values for the reconstruction, the UBD-based method substitutes this step with an endmember extraction step, finding a set of reference pixels which are optimal at representing any image element as their combination. If we consider that each endmember is naturally related to a class of interest as selected in [8], this approach can be regarded as a way of selecting the most suitable pixel among the ones assigned to a given class, improving the accuracy in reconstruction. Furthermore, nonnegativity constraint is enforced in the inversion step: this was shown to be effective at improving the reconstruction results, as negative contributions from the reference image elements have no physical meaning [2].

\section{EXPERIMENTAL RESUlts}

We analyse a $500 \times 500$ subset of a SITS stack composed of 9 multispectral RapidEye images, with 5 spectral bands and a spatial resolution of 5 meters, acquired over agricultural fields in the north of Israel in a time span of 9 months (October 2013 to July 2014). An RGB of an image acquired in June 2014 with a cloud/shadow coverage of approximately $55 \%$ is reported in Fig. 3(a), while all other images belonging to the stack are cloud-free. From the common cloud-free area 30 pixels are collected: afterwards, a spectral unmixing procedure is carried out on all the images but the cloudy one through Non-Negative Least Squares (NNLS) [1] following eq. (1). The computed abundances are then used to reconstruct the pixels in the area of interest (covered by clouds or their shadows) using eq. (2). Results are reported in Fig. 3(b). The transition between cloud-free pixels and reconstructed regions is seamless. It is remarkable that, instead of selecting pixels from other cloud- 


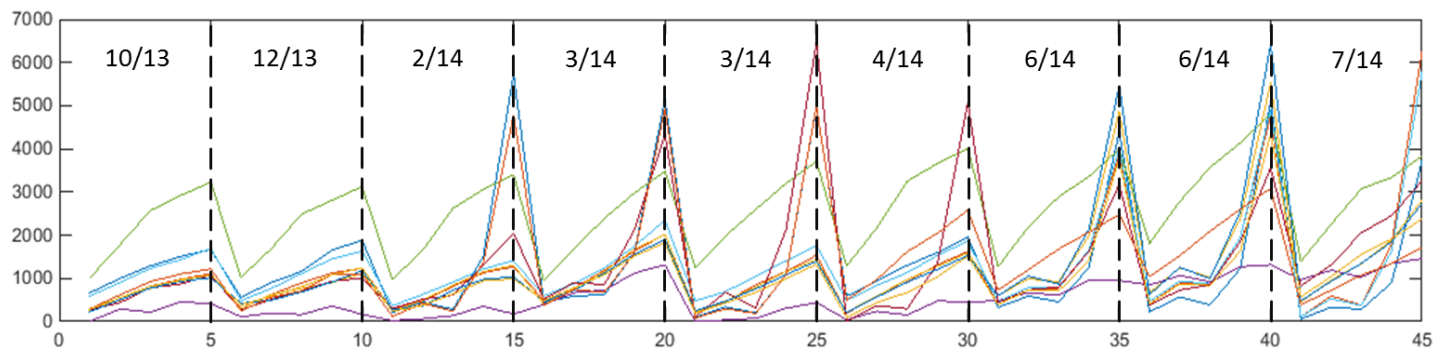

Fig. 1: Sample basis vectors for the synthesis of pixels covered by clouds. The evolution in time of 10 image elements such as the ones selected in Fig. 2 (a) is captured across the nine images in the stack, each composed by five spectral bands. Dashed lines mark the transitions between different images in the stack.

free images in the stack, the reconstructed area is obtained by rearranging the spectral information from cloud-free pixels in the same image of interest. This is achieved exploiting the information on the pixels composition derived from the other images in the stack.

\section{CONCLUSIONS}

This paper presents a novel algorithm for cloud removal based on the idea of spectral unmixing, a technique usually applied to hyperspectral datasets to decompose single image elements in fractional abundances of spectra related to macroscopically homogeneous materials present in the scene. It is possible to perform the same decomposition on pixels in SITS, where the basis vectors are cloud-free pixels exhibiting characteristic evolutions in time. After the decomposition using only the cloud-free images in a SITS, a cloudy pixel is reconstructed as a weighted sum of the values of the basis vectors in the image it belongs to. Results show that the reconstructed areas blend seamlessly in the image and results could be similar to the values expected by an user. The main advantage of this method with respect to traditional ones, which select cloud-free pixels from other images in the stack, is that it estimates the value of a pixel covered by clouds by manipulating other values in the same image. This is possible also if only one image is available for a given period of time, or if the image of interest is radiometrically different due to characteristic atmospheric absorption phenomena. The drawback of the method is that it makes the assumption that it is possible to locate cloud-free samples for each object covered by the clouds which evolves in the same way through time. In the future the algorithm could be fully automatized by including a cloud and shadow mask generation, and by running selected VD estimation and endmember extraction algorithms (usually employed in applications to hyperspectral data) prior to the abundance estimation and pixel reconstruction steps.

\section{ACKNOWLEDGMENTS}

The authors would like to thank the German Aerospace Center (DLR), RESA Science Team, Neustrelitz for the support for providing the satellite data of the RapidEye Science Archive (proposal no. 597). We also thank Thomas Krauss and Peter Fischer (DLR) for the atmospheric correction of the images and Thomas Jarmer and Florian Beyer (University of Osnabrueck) for compiling the satellite image time series.

\section{REFERENCES}

[1] J. M. Bioucas-Dias, A. Plaza, N. Dobigeon, M. Parente, Q. Du, P. Gader, and J. Chanussot, "Hyperspectral unmixing overview: Geometrical, statistical, and sparse regression-based approaches," IEEE Journal of Selected Topics in Applied Earth Observations and Remote Sensing, vol. 5, no. 2, pp. 354-379, 2012.

[2] D. Cerra, R. Müller, and P. Reinartz, "Noise reduction in hyperspectral images through spectral unmixing," IEEE Geoscience and Remote Sensing Letters, vol. 11, no. 1, pp. 109-113, Jan 2014.

[3] D. Cerra, R. Müller, and P. Reinartz, "Unmixing-based denoising for destriping and inpainting of hyperspectral images," in IGARSS 2014, July 2014, pp. 4620-4623.

[4] D. Cerra, J. Bieniarz, T. Storch, R. Müller, and P. Reinartz, "Restoration of enmap data through sparse reconstruction," in Workshop on Hyperspectral Image and Signal Processing: Evolution in Remote Sensing (WHISPERS), 2015, June 2015, pp. 1-4.

[5] O. Hagolle, M. Huc, D. Villa Pascual, and G. Dedieu, "A multi-temporal method for cloud detection, applied to FORMOSAT-2, VENS, LANDSAT and SENTINEL-2 images," Remote Sensing of Environment, vol. 114, no. 8, pp. 1747-1755, Aug. 2010.

[6] A. Makarau, R. Richter, R. Muller, and P. Reinartz, "Adaptive shadow detection using a blackbody radiator model," Geoscience and Remote Sensing, IEEE Transactions on, vol. 49, no. 6, pp. 2049-2059, June 2011.

[7] Chein-I Chang, Chao-Cheng Wu, and Ching-Tsorng Tsai, "Random $\mathrm{n}$-finder (n-findr) endmember extraction algorithms for hyperspectral imagery," IEEE Transactions on Image Processing, vol. 20, no. 3, pp. 641-656, 2011.

[8] F. Melgani, "Contextual reconstruction of cloud-contaminated multitemporal multispectral images," IEEE Transactions on Geoscience and Remote Sensing, vol. 44, no. 2, pp. 442-455, Feb 2006. 


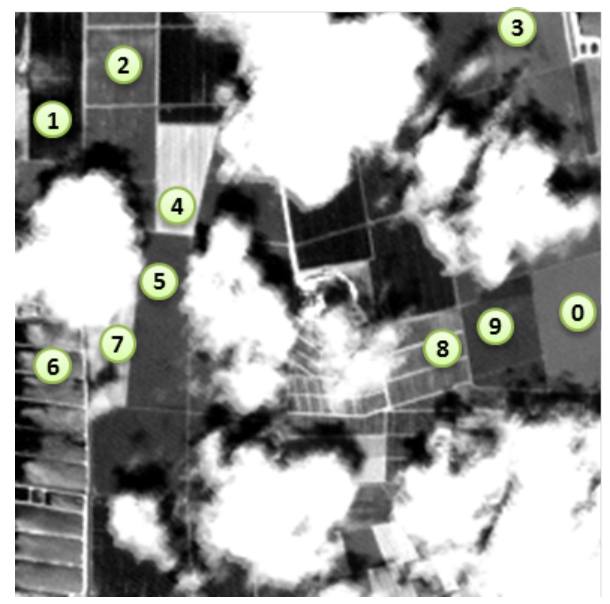

(a) Selection of cloud-free representative pixels, here numbered from 0 to 9 .

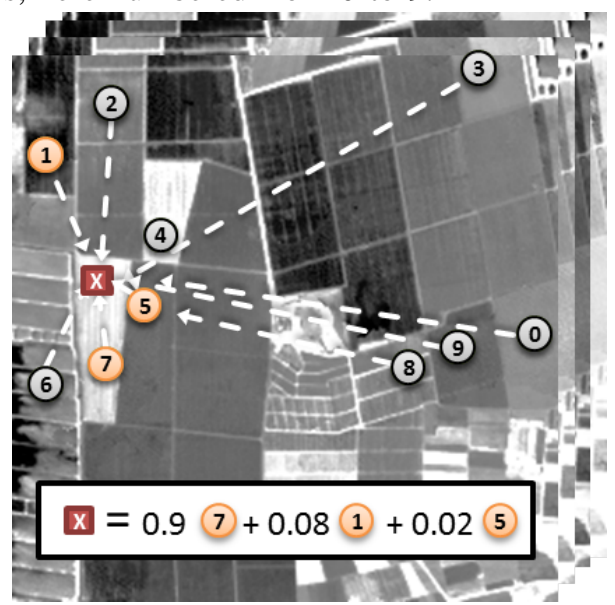

(b) Decomposition of an image element of interest (the $x$ in the red square) in terms of the selected pixels, computed using all the available cloud-free images in the stack.

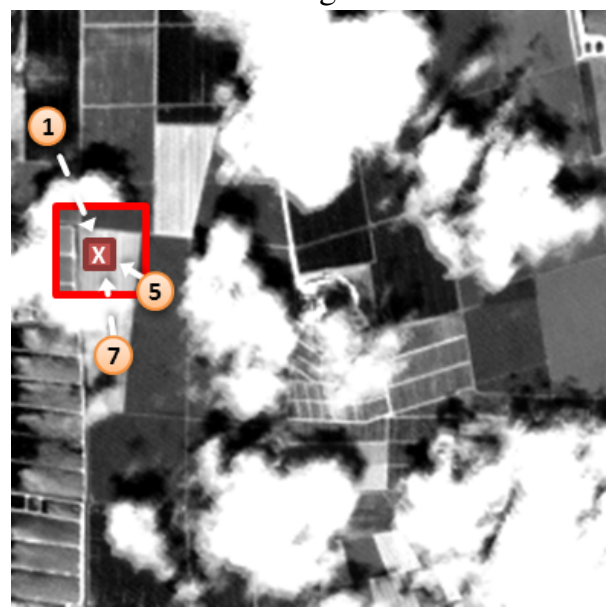

(c) The pixel of interest and its neighbours are reconstructed using cloud-free pixels from the same image as basis vectors.

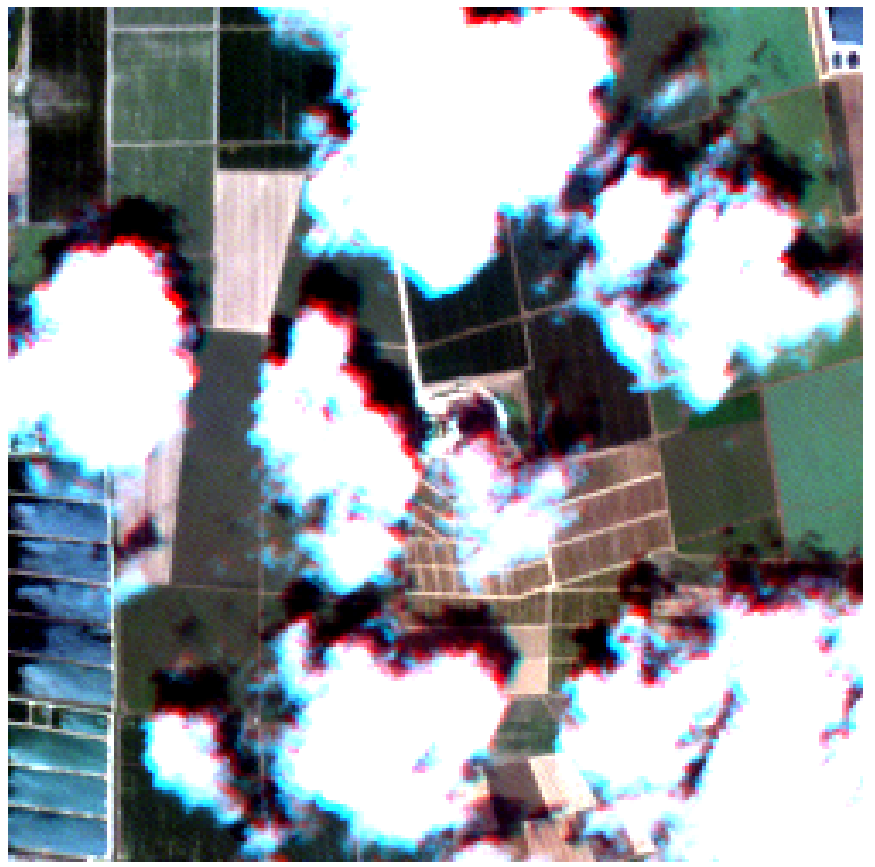

(a) RGB composite of cloudy image.

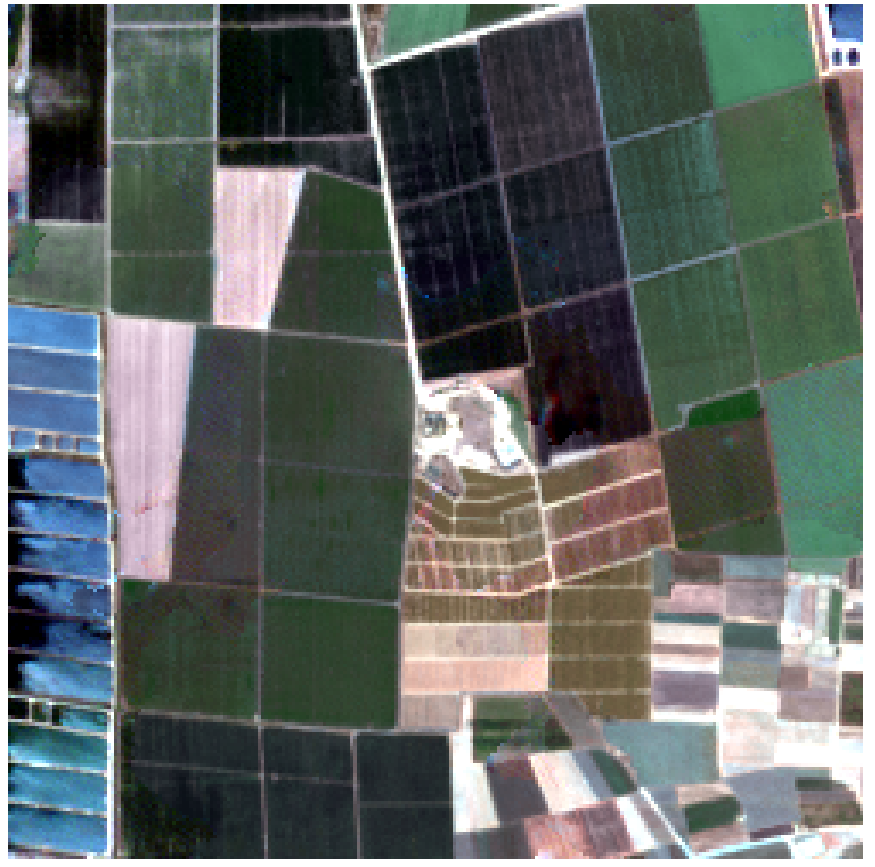

(b) Results of the proposed cloud removal algorithm.

Fig. 3: Experimental results.

Fig. 2: Workflow for the estimation of the expected values of image elements covered by clouds (or their shadow). 